\title{
AC MAGNETIC FIELD FREQUENCY DEPENDENCE OF MAGNETOACOUSTIC EMISSION
}

\author{
M. Namkung, B. Wincheski ${ }^{\dagger}$, J. P. Fulton ${ }^{\dagger}$, and R. DeNale , $^{\dagger}$ \\ NASA Langley Research Center, Hampton, VA 23665 \\ ${ }^{\dagger}$ Analytical Services and Materials, Inc., 107 Research Dr., Hampton, VA 23666 \\ ${ }^{\dagger \dagger}$ Caderoc Division of Naval Surface Warfare Center, Annapolis, MD 21402
}

\begin{abstract}
Our recent study has proved a strong correlation between the low-frequency AC applied magnetic field amplitude dependence of the asymmetry of the magnetoacoustic emission (MAE) burst and the strength of the domain wall-defect interaction in iron-base ferromagnets. For the present study the AC magnetic field frequency dependence of the asymmetry has been investigated in the range of 1 to $200 \mathrm{~Hz}$. When represented by the third moment of the rectified acoustic emission pulses, the asymmetry becomes a bell-shaped function of frequency with its center located around $25 \mathrm{~Hz}$. This experiment has been performed with low carbon, high yield stress steel specimens of three different levels of domain wall-defect interaction strength. The results show that the increase in the interaction strength causes a vertical down shift of the asymmetry in the entire frequency range investigated.
\end{abstract}

\section{INTRODUCTION}

Certain material characteristics originate from properties which are localized in a scale much smaller than the resolution limit of most nondestructive evaluation (NDE) techniques. A typical example is temper embrittlement in low carbon steel with high yield stress which is caused by the trapping of impurities such as sulphur, phosphorous, tin, antimony and arsenic in the grain boundaries during the post heat treatment after casting [1]. The high density of these elements weakens the mechanical strength of the grain boundaries significantly reducing the impact resistance of the material. Nevertheless, other static material properties such as elastic modulus and tensile strength remain unaffected by temper embrittlement [1].

The MAE technique is known to provide detailed information on the local interaction between grain boundaries and magnetic domain walls during the repeated changes in the magnetic states induced by an externally applied AC magnetic field [1,2]. It was also found that an ascending asymmetry in the MAE burst, formed by a train of individual pulses observed for every half cycle of the hysteresis loop, is caused by embrittlement, whereas a descending asymmetry is caused by a uniaxial compressive stress applied parallel to the AC magnetic field $[3,4]$. The former is based on the domain wall-defect interaction while the latter is based on the compressive stress-induced anisotropic viscosity of magnetic domain walls.

In our recent study the asymmetry in the MAE bursts was parameterized as the skewness from the spectrum averaged over 50 half cycles through the hysteresis loop and its dependence on the amplitude of the applied AC magnetic field at $0.7 \mathrm{~Hz}$ has been investigated [5]. The skewness was found to be a decreasing function of the AC field amplitude, and the probability of separating the unembrittled and embrittled specimens was seen to be enhanced by this method. The present study is to investigate the effect of the AC applied magnetic field frequency variation on the skewness to study whether an even clearer separation of embrittled samples can be accomplished.

\section{EXPERIMENTS AND NUMERICAL PROCESSING}

Three low carbon steel samples were selected for the present study. The first sample has never been post heat treated and is unembrittled. The second sample has been heat treated for 1 hour and the third one has been heat treated for 50 hours at $538^{\circ} \mathrm{C}$ after casting (see Ref. 1 for details). The impact strength of the these samples are estimated to be about 210, 89, and 11 newton-meters (127, 54 and $6.5 \mathrm{ft-Lbs)}$ respectively. Details of the experimental setup can be found elsewhere $[3,4]$ so that only the numerical processing procedure will be fully described.

Due to the random nature involved in the continuous cycles of magnetic domain wall recreationannihilation over the hysteresis loops, it is nec- 
essary to average the MAE spectra obtained for a certain period. The digitized signals of MAE spectrum were rectified and summed for 50 half cyles of the hysteresis loop and the skewness was calculated from the resultant spectrum as follows.

The first step is to construct a normalized distribution from the rectified and averaged MAE spectrum which is defined as

$$
g\left(t_{i}\right)=\frac{h\left(t_{i}\right)}{\sum_{j}^{N} h\left(t_{j}\right)}
$$

where $h\left(t_{i}\right)$ represent the average rectified individual pulses of the raw MAE spectrum. $g(t)$ was then smoothed and the first moment was obtained as

$$
E(t)=\mu_{t}=\sum_{i}^{N} t_{i} g\left(t_{i}\right)
$$

which is the weighted average of the points $t_{i}$. The variance is then defined as

$$
E\left(t-\mu_{t}\right)^{2}=\sigma_{\tau}^{2}=\sum_{i}^{N}\left(t_{i}-\mu_{t}\right)^{2} g\left(t_{i}\right)
$$

which is a measure of the spread of $g(t)$ about its mean. The skew is then defined as follows:

$$
S k_{t}=\sum_{i}^{N} \frac{\left(t_{i}-\mu_{t}\right)^{3} g\left(t_{i}\right)}{\left(\sigma_{\tau}^{2}\right)^{\frac{3}{2}}}
$$

The frequency of the $\mathrm{AC}$ applied magnetic field was varied from 1 to $200 \mathrm{~Hz}$ and incremented differently over various ranges of frequencies. The signal-to-noise ratio decreases significantly at high frequencies. Hence, for the sake of clarity and without losing any meaningful informaton, the experimental results were presented up to $50 \mathrm{~Hz}$.

The power supply output was controlled by the sinusoidal signals from a function generator which has been maintained at a constant amplitude throughout the entire experiment. The actual AC magnetic field amplitude in the samples, of course, varied upon frequency change. To establish a reference, the peak AC magnetic field amplitude at the surface was measured using a Chattock-type magnetic potentiometer [6]. The potentiometer was fabricated by winding 450 turns of 36 gauge wire on a semicircular-shaped plastic core of $25.4 \mathrm{~mm}$ diameter and approximately $4 \times 5 \mathrm{~mm}^{2}$ cross-section. For calibration a sinusoidal AC magnetic field was produced by a helmholtz pair, and the linear relationship between the peak amplitudes of integrated potentiometer output and the AC magnetic field has been established. The purpose of integration was to shift the entire waveform such that the peak amplitude of magnetic field at the surface of the sample can be measured while satisfying $d \phi / d t=0$ which is the condition for a vanishing surface current where $\phi$ is the total magnetic flux. Hence, the peak tangential component of the magnetic field immediately outside of the surface was the same as that immediately inside the surface of the sample [5]. The results of these measurements are shown in Fig. 1.

\section{RESULTS AND DISCUSSION}

Fig. 2 (a) shows the output of the induction pickup coil and (b) shows the raw MAE spectrum obtained with the untreated sample at $.7 \mathrm{~Hz}$. The rectified and averaged MAE spectrum is shown in Fig. 2 (c) and the spectrum was further processed by smoothing as seen in Fig. 2 (d). The skewness was then computed from the final form of the MAE spectrum as described in the previous section.

Fig. 3 shows the pickup coil output and the processed MAE spectrum obtained with the sample heat treated for 50 hours. One can immediately notice the difference between the MAE spectra of Fig. 2 (d) and Fig. 3 which is due to the effect of the enhanced magnetic domain wall-defect interaction.

As is well known, the $90^{\circ}$ domain walls in ironlike ferromagnets are mainly responsible for the generation of MAE [3,4]. With higher concentration of impurities the grain boundaries of the embrittled specimen become stronger pinning sites. The motion of $90^{\circ}$ domain walls requires a stronger driving force which is only achieved at a later phase of the AC applied field cycle. Hence the peak MAE amplitude in an embrittled sample appears

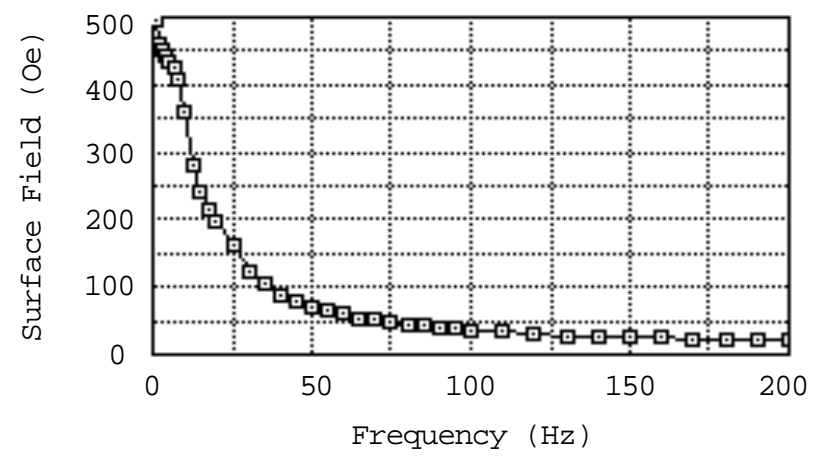

Fig. 1. Peak amplitude of surface magnetic field as a function of AC applied magnetic field frequency. 


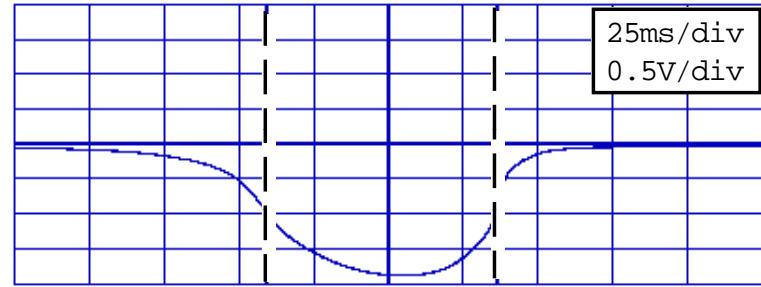

(a) induction pickup coil

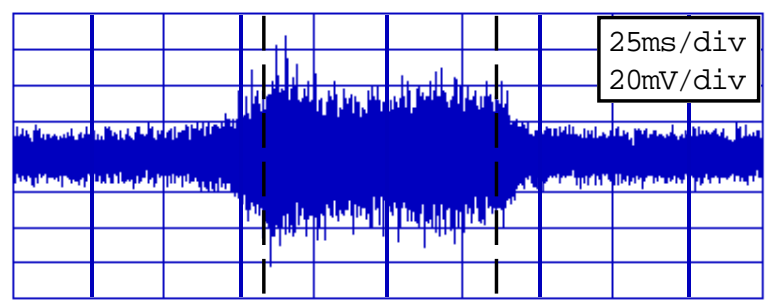

(b) unprocessed MAE waveform

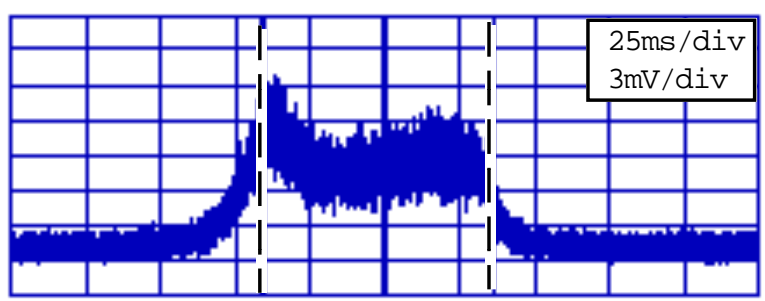

(c) rectified and averaged waveform

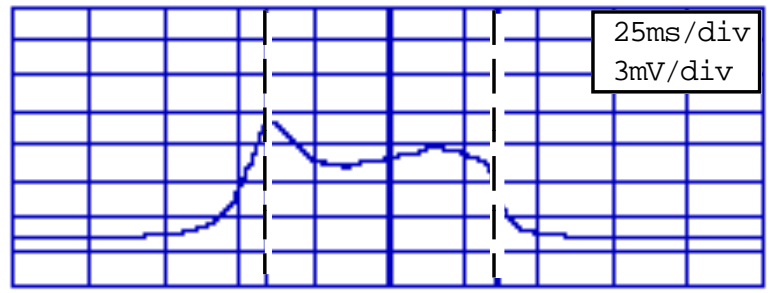

(d) rectified, averaged and smoothed waveform

Fig. 2. Pickup coil output and MAE spectrum of the untreated sample at $0.7 \mathrm{~Hz}$

later in time compared with that of an unembrittled sample.

The lower and upper limits of processing were selected by finding the maximum slope in the pickup coil signal for the frequency up to $15 \mathrm{~Hz}$. The skew was computed for the three samples and is shown in Fig. 4. Apparently, the frequency dependence of the skew is almost identical for all three samples except that the curves are separated vertically. Such an initial increase in the skew can be explained by an accelerated motion of $90^{\circ}$ domain walls as the rate of change in the flux becomes more rapid.

Fig. 5 shows the pickup coil output and the processed MAE spectrum obtained with the $50 \mathrm{Hr}$. heat treated sample at $25 \mathrm{~Hz}$. As is seen in Fig. 1, the
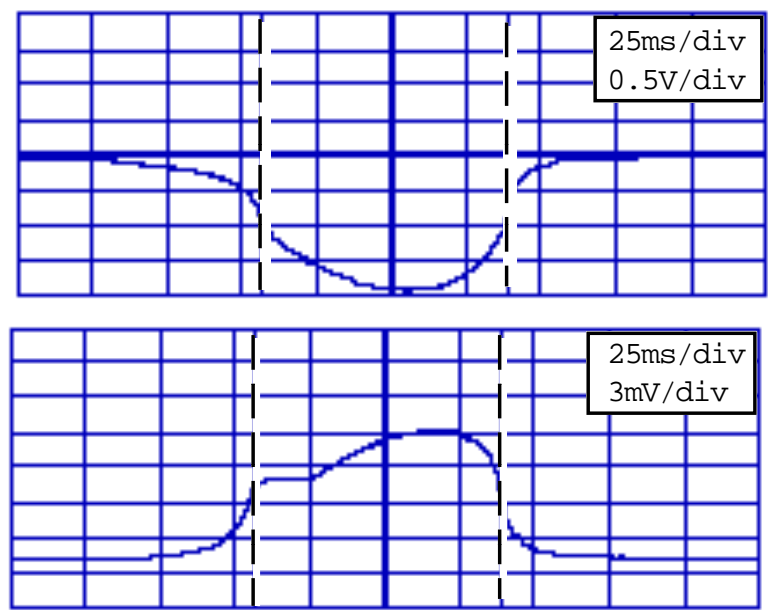

Fig. 3. Pickup coil output and MAE spectrum of the $50 \mathrm{hr}$. heat treated sample at $0.7 \mathrm{~Hz}$

magnetic field strength in this frequency range is considerably lower and the B-H sweep occurs along a minor hysteresis loop causing a much different shape of the pickup coil output. Hence, the lower/ upper limits of the processing region were selected from the minima of the MAE spectrum obtained for one half cycle of the hysteresis loop.

Fig. 6 and Fig. 7 show the results of the skews for the frequency ranges of $15-30 \mathrm{~Hz}$ and $30-50 \mathrm{~Hz}$, respectively. The skew changes from an increasing to a decreasing function at approximately $22 \mathrm{~Hz}$. Such a reversal in slope is directly related to the limit of mobility of the $90^{\circ}$ domain walls in response to the externally applied AC magnetic field. It is, however, unexpected that the overall AC magnetic field frequency dependence of the skew is independent of the domain wall-defect interac-

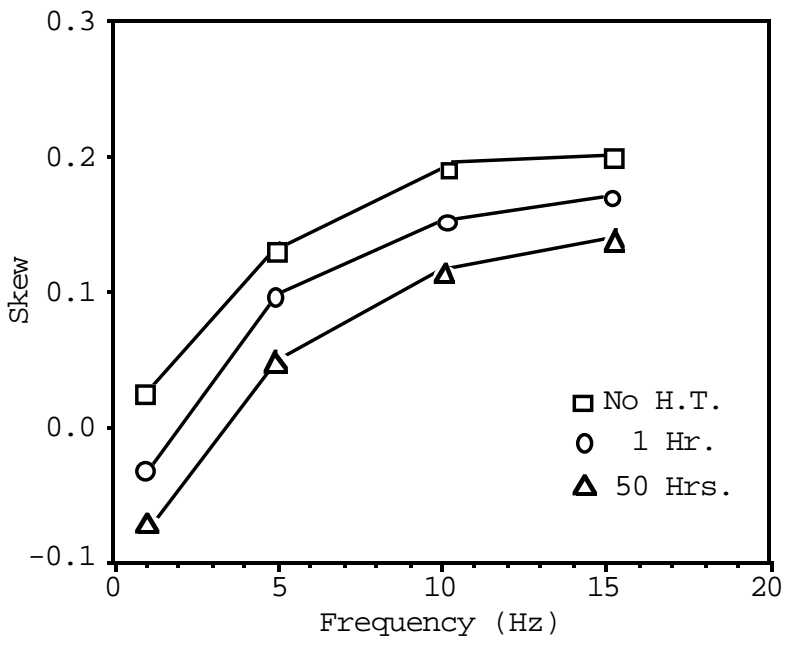

Fig. 4. Frequency dependence of skew in low frequency region 

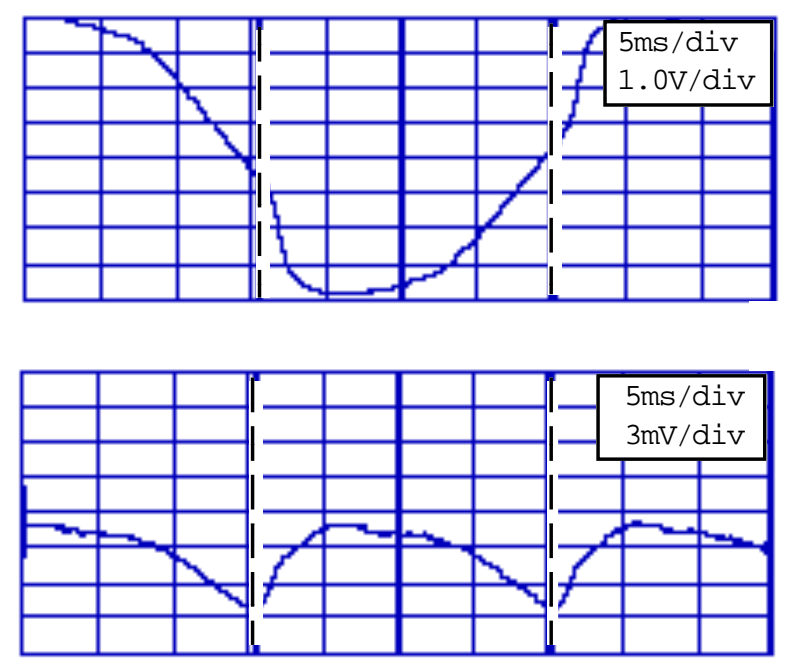

Fig. 5. Pickup coil output and MAE spectrum of the $50 \mathrm{hr}$. heat treated sample at $25 \mathrm{~Hz}$

tion strength except for a separation along the vertical axis. These results may confirm that there exists a certain fraction of $90^{\circ}$ domain walls involved in MAE making a frequency independent contribution to the skew. Nevertheless, it is clear that varying the AC magnetic field frequency does not add any new capability for differentiating between the embrittled and unembrittled samples. Further, the present results justify our original decision of choosing a very low AC magnetic field frequency $(0.7 \mathrm{~Hz})$ for the MAE embrittlement characterization $[3,4]$.

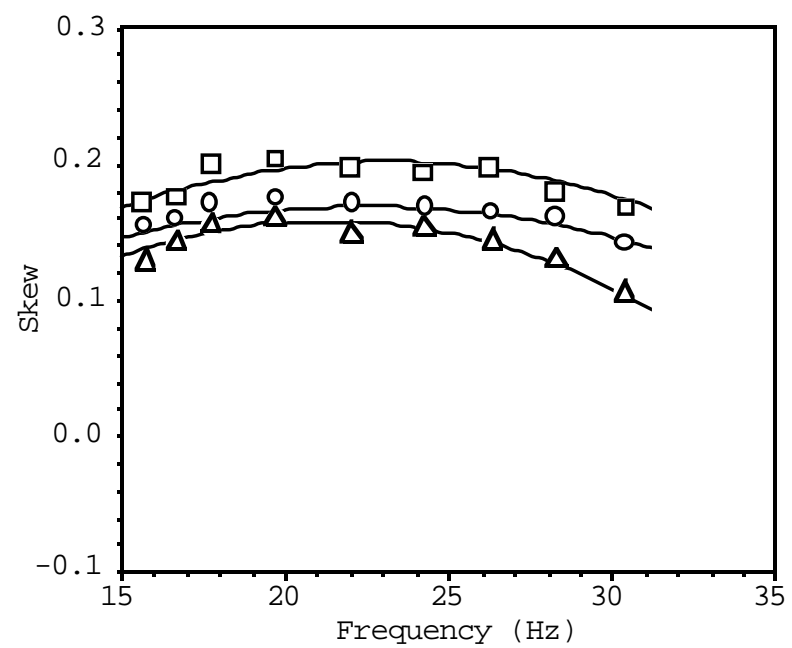

Fig.6. Frequency dependence of skew in intermediate frequency region

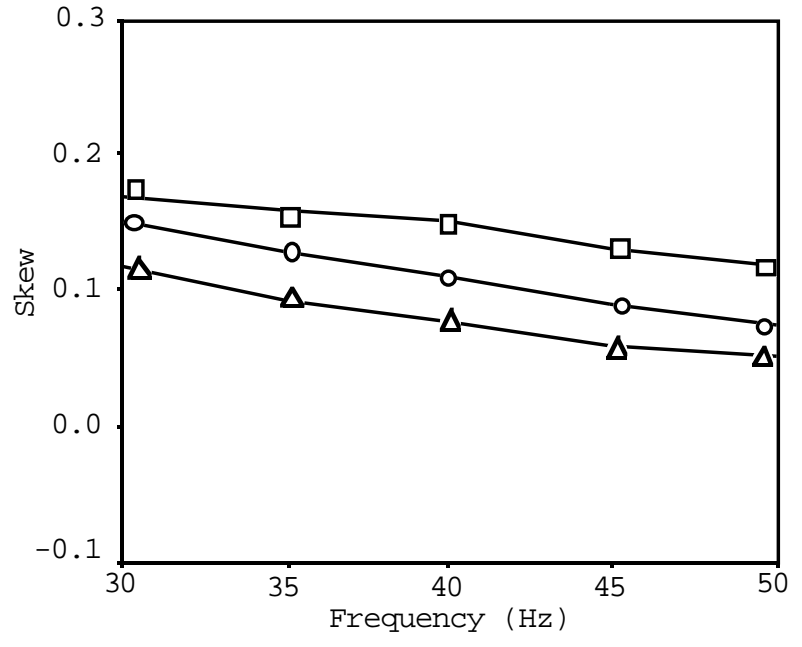

Fig. 7. Frequency depencence of skew in high frequency region

\section{SUMMARY}

A series of experiments were performed to investigate the AC applied magnetic field frequency dependence of skew of three low carbon steel samples with varying degrees of domain wall-defect interaction strength. The skew is found to increase initially, reaches a maximum at about 22 $\mathrm{Hz}$, and begins to decrease with further increases in the AC applied magnetic field frequency. This result is explainable, such a trend being independent of the interaction strength is, however, unexpected and needs to be studied more systematically for a better understanding of the basic properties of ferromagnets.

\section{REFERENCES}

[1]. S. G. Allison, W. T. Yost, J. H. Cantrell, $\mathrm{Jr}$. , and D. F. Hasson, Review of Progress in QNDE, Vol. 7B, 1463 (Plenum Press, New York, 1988). [2]. M. Namkung, W. T. Yost, D. Utrata, J. L. Grainger and P. W. Kushnick, Proc. IEEE Ultrasonics Symposium, Vol. 2, 983 (1991).

[3]. M. Namkung, R. DeNale and R. G. Todhunter, Review of Progress in QNDE, Vol. 10B, 2007 (Plenum Press, New York, 1991).

[4]. M. Namkung, D. Utrata and R. DeNale, Proc. IEEE Ultrasonics Symposium, Vol. 2, 983 (1990). [5]. M. Namkung, B. Wincheski, J. P. Fulton and R. DeNale, submitted to Review of Progress in QNDE, LaJolla, CA (July 19 - 24, 1992).

[6]. L. F. Bates, Modern Magnetism (Cambridge Press, 1963). 
